\title{
The "thin man" phenomenon: a sign of cortical plasticity following inferior homonymous paracentral scotomas
}

\author{
Avinoam B Safran, Olivier Achard, Florence Duret, Theodor Landis
}

Neuro-ophthalmology and Strabology Unit, Ophthalmology Clinic, Department of Clinical Neurosciences, Geneva University Hospitals, Geneva, Switzerland A B Safran

O Achard

F Duret

Neurology Clinic, Department of Clinical Neurosciences, Geneva University Hospitals, Geneva, Switzerland T Landis

Correspondence to: Avinoam B Safran, MD, Neuro-Ophthalmology and Strabology Unit, Ophthalmology Clinic, Department of Clinical Neurosciences, Geneva University Hospitals, CH-1211 Geneva 14, Switzerland

Accepted for publication 30 July 1998
Abstract

Aim-To investigate an image distortion, experienced by patients with homonymous paracentral scotomas.

Methods-Two consecutive patients with right inferior homonymous paracentral scotomas resulting from ischaemic brain insults were examined. Neuroophthalmological examination included tangent screen and Amsler grid evaluation. In addition, the patients were asked to describe a figure showing two vertical lines, identical in length and symmetrically located on either side of a fixation point. This figure was presented in such a way that when the subject looked at the fixation point the right line crossed the scotoma. Finally, the patients were asked whether, when looking at the face of an interlocutor, both sides of the body looked the same.

Results-In both patients field defects were markedly smaller when delineated with Amsler grids than using a tangent screen. With the parallel line test, the right line appeared uninterrupted in patient 1 , whereas in patient 2 it looked slightly blurred in a two degree long segment corresponding to the middle of the scotoma. To both subjects the right line appeared shorter than the left line. Finally, both subjects indicated that, after steadily fixating their interlocutor's face or neck for 5-10 seconds, the left shoulder appeared narrower than the right one, which made him look surprisingly thin. This perceptual alteration was called the "thin man" phenomenon.

Conclusions-Paracentral homonymous scotomas can be associated with perceptual completion and shape distortion, owing to apparent displacement of images adjacent to the scotoma towards the field defect. Occurrence of such a perceptual change should alert one to the possibility of paracentral homonymous scotomas, which often go undetected when using routine visual field testing procedures.

(Br f Ophthalmol 1999;83:137-142)
It has recently been shown that, in adults, maps in the cerebral cortex are not fixed. ${ }^{1}$ Instead, receptive fields of cortical neurons can reorganise following deactivation or an altered pattern of activation. As a result of cortical plasticity, localised defects of the visual field generate a filling in of the scotomas, ${ }^{2}$ and a spatial distortion of images in the field surrounding the scotoma. ${ }^{34}$ Filling in causes affected subjects to underestimate or ignore their defects, whereas spatial distortion is manifested by objects lying near the scotoma borders as appearing pulled towards the centre of the visual field defect.

In two successive patients with right inferior homonymous paracentral scotomas, we noted the occurrence of a striking form of object distortion that probably reflected both filling in and spatial distortion. The first patient volunteered the information that, when speaking with someone, her interlocutor's left shoulder appeared to be narrower than normal, which

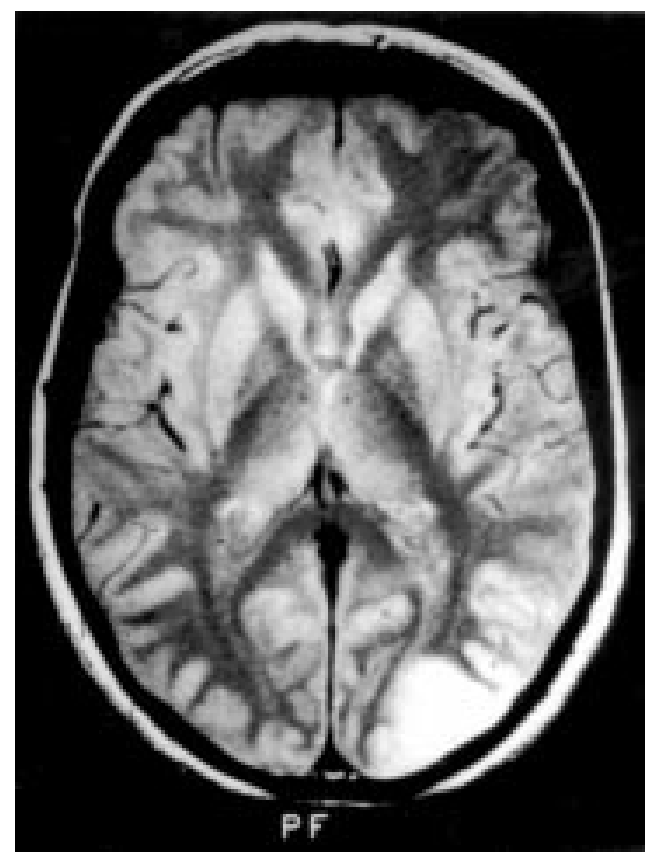

Figure 1 Cerebral magnetic resonance image of patient 1 , showing, on a T2 weighted image, a left sided lesion extending from the occipital pole into the lateral occipital gyri. 


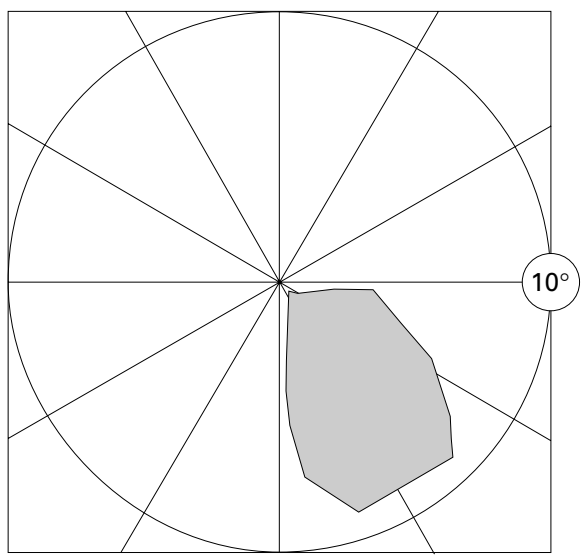

LE

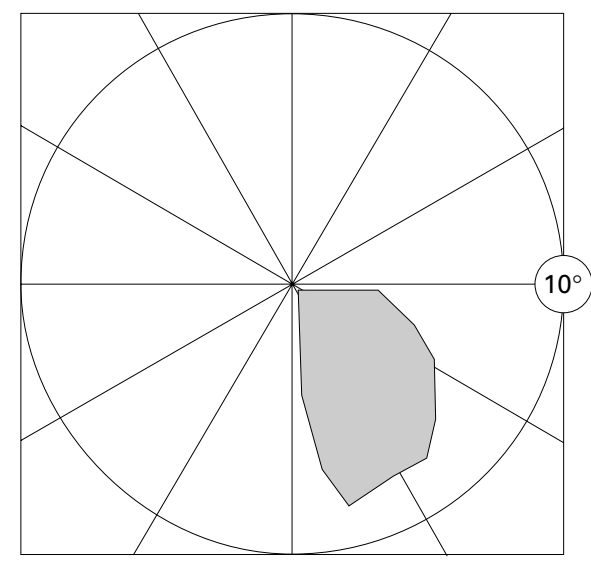

$\mathrm{RE}$

$\square 2 \mathrm{w} / 1000$

Figure 2 Right paracentral homonymous scotoma of patient 1, delineated by tangent screen.

made him look surprisingly thin. Following this observation, in a carefully unbiased manner, we asked our next patient showing similar visual field defects whether, when looking at the face of an interlocutor, he observed an unexpected alteration in body proportions. To his own astonishment, he clearly described an identical change in perception to that reported by our first patient.

We named this visual phenomenon the "thin man" phenomenon.

\section{Patients and methods}

The medical history and the clinical findings of the two patients were described as follows.

The first patient was a 50 year old woman with a long history of common migraine, who suffered an episode of intense, diffuse, throbbing headache. The pain was associated with nausea and photophobia, and lasted 24 hours. During this period, the patient remained lying down in a darkened room. When the pain had gone, she noticed difficulty in reading, with words appearing to be truncated. A neurological examination showed a right paracentral homonymous defect. Blood tests for metabolic alterations associated with vascular occlusions or pseudovascular conditions, including mitochondrial abnormalities, and extensive cardiovascular investigations, including cervical and transcranial Doppler evaluation, were all normal. In contrast, cerebral magnetic resonance imaging (MRI) showed, on both $\mathrm{T} 1$ and $\mathrm{T} 2$ weighted images, a lesion situated at the left occipital pole, extending laterally into the occipital gyri (Fig 1).

A neuro-ophthalmological evaluation was performed 3 days later, and the scotoma was delineated using a 1 metre distant tangent screen (Fig 2). Visual acuity was normal, but when asked to read sentences the patient noted that parts of words were often missing. When missing letters were located in the middle of a word, the initial and final letters appeared to be contiguous with no gap in between, and thus seemed to constitute an uninterrupted word. Refraction and orthoptic evaluations were unremarkable.
Interestingly, 1 week after the onset of the disorder, the patient volunteered that, when looking at the face or at the neck of an interlocutor, his left shoulder appeared much smaller than his right one. She also noted that the lower left side of his face seemed slightly contracted. She added that no parts of her interlocutor's body appeared to be missing, but that affected areas of the visual field caused certain parts of his body to appear smaller. Images adjacent to the scotoma were displaced towards the area of the field defect.

The second patient was a 62 year old man, with a history of hypercholesterolaemia and arterial hypertension. He was hospitalised for an ischaemic event in the territory of the left posterior cerebral artery, with associated loss of vision in the right homonymous hemifield. On both $\mathrm{T} 1$ and $\mathrm{T} 2$ weighted images, cerebral MRI demonstrated a small lesion located at the left occipital pole (Fig 3), and two other small lesions situated more anteriorly and more dorsally at the occipitoparietal junction.

Three months after the onset of the disorder, his visual fields showed a right inferior

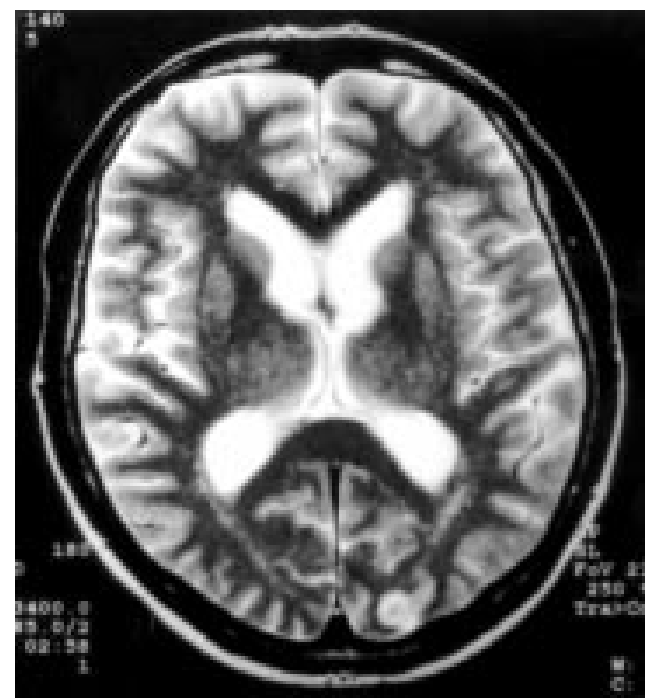

Figure 3 Cerebral magnetic resonance image of patient 2, showing, on a T2 weighted image, a single small lesion at the left occipital pole. 


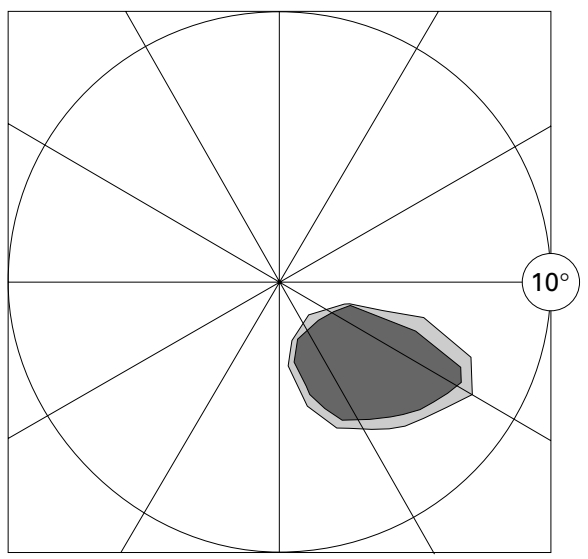

LE

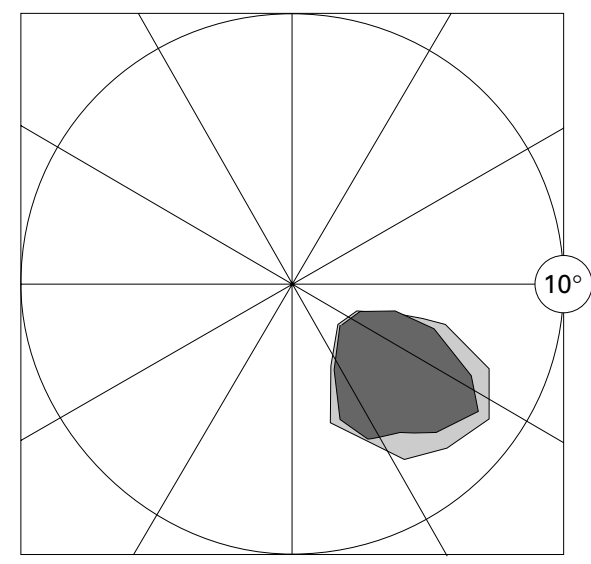

RE

$10 \mathrm{w} / 1000 \square 2 \mathrm{w} / 1000$

Figure 4 Right paracentral homonymous scotomas of patient 2, delineated by tangent screen.

homonymous paracentral scotoma, when delineated using a 1 metre distant tangent screen (Fig 4).

To characterise further the perception of the defect by the affected patients, they underwent additional tests. Firstly, they were asked to carefully describe a white on black Amsler grid with both eyes open. Secondly, they were shown a white sheet of paper, on which two parallel vertical black lines were drawn on either side of a small fixation point. The lines extended at 5 degrees above the upper border, and about 5 degrees below the lower border of the scotoma (Fig 5). Patients were asked to describe the figure, and in particular, to comment the relative lengths of the lines. The vertical lines were shown at a distance of approximately 5 degrees from the fixation point, on either side of it, with the first patient, and of approximately 4 degrees with the

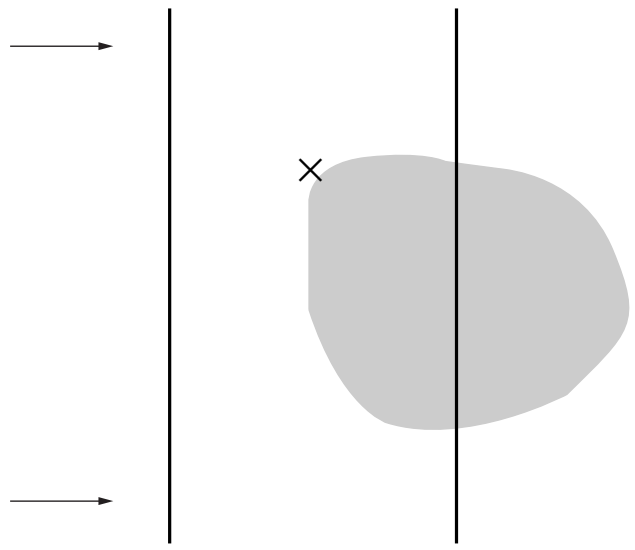

Figure 5 Schematic representation of the parallel line test evaluating the completion phenomenon in subjects with a paracentral scotoma. It shows the relative positions of the fixation point (cross), vertical test lines, and right paracentral scotoma (shaded area). The vertical lines are equal in length and are equidistant from the fixation point. They are presented to the subject in such a way that the line located on the affected side of the visual field crosses the scotoma, and extends for a short distance on either side of $i$. After steadily fixating the cross for 5-10 seconds, the subject is asked about any perceived interruption in the lines or inequality in their length, and is asked to indicate, on the line located on the unaffected side, the levels corresponding to the perceived upper and lower ends of line on the affected side (as exemplified by the two arrows). second patient. Finally, the second patient was asked whether, when looking at his interlocutor's face, both sides looked the same.

\section{Results}

When asked to describe the Amsler grid, the first patient indicated that she did not observe either interruptions or other forms of localised alterations in the grid pattern. Using the test with two parallel lines, she reported that both lines appeared uninterrupted, but that the right line appeared to be shorter than the left one. The patient indicated this subjective difference in the length of the lines using a pen to show on the left line where the ends of the right line appeared to be; she pointed about one degree below the upper end and one degree above the lower end of the left line. This difference in length of the lines appeared distinctly only after several seconds of observation.

Using the Amsler grid, the second patient delineated an area of attenuated grid pattern that was approximately 4 degrees in height and 6 degrees in width. When examined using the test with two parallel lines, he indicated that while the left line was unremarkable the right line was slightly blurred in an approximately 2 degree long segment, which corresponded to the middle of the scotoma area. When asked about the relative lengths of the lines, he reported that the left line extended slightly further than the right line, at both its upper and lower ends, by a distance which was estimated to be slightly less than one degree at each end. The difference in length of the lines was much more evident after several seconds of observation.

When the patient was asked whether, when looking his interlocutor's face, both sides looked the same, he replied that the lower left part of the face, seen in his right hemifield, was slightly altered, but the changes were difficult to describe. He also stated that the left shoulder of the person facing him - that is, the shoulder seen in his right hemifield, appeared distinctly narrower than the other one. $\mathrm{He}$ observed no other apparent defect in the shoulder. 


\section{Discussion}

Our patients showed homonymous paracentral scotomas that were inferior in location, presumably resulting from ischaemic insults. These patients did not perceive the visual field defects as gaps. Nor, when looking at their interlocutor's face, did any part appear to be missing except, in the affected hemifield, for a contraction phenomenon in the shoulder in comparison with the other side. This made the person appear thinner and somewhat distorted.

There are several reasons to believe that asymmetry in the perceived image was related to phenomena induced by cortical plasticity, including filling in and spatial distortion. ${ }^{5}$

In these patients, occurrence of filling in-that is, a perceptual interpolation that allows visual stimuli to be perceived where there is actually no visual input, was demonstrated by the fact that no gaps was perceived in their visual field. ${ }^{6}$ Filling in was also shown by the use of Amsler grids, and by the parallel line test which we devised for this study. With the former, the patients reported either the absence of alteration in the grid pattern, or the perception of an area of alteration that was much smaller than the surface of the scotoma delineated by perimetry. This phenomenon is similar to that observed when using Amsler grids in patients with central scotomas due to macular disorders. ${ }^{7}$ It should alert us to the limitations of assessing scotomas by methods relying on the subject's perception of the defect against a structured background, such as that of an Amsler grid. ${ }^{5}$

Perceptual changes experienced by our patients were also related to spatial distortion. This was apparent in the first subject, when letters were missed in the middle of a word due to the homonymous scotoma, the initial and final letters being perceived as contiguous and constituting a single, complete word.

Moreover, distortion was demonstrated with the parallel line test, the right line, crossing the scotoma, appearing shorter than the left one. Although the perceived asymmetry was moderate in relation to the scotoma it was clearly observed by both our patients. This form of shortening obviously was different from the condition in which one end of the line is projected into the scotoma; in such a case one peripheral segment of the line is simply occult, and the changes in the perceived length of the line are pronounced.

Interestingly enough, the difference in length was apparent only after several seconds of image analysis. This delay is in accordance with findings of previous studies, which showed that the mechanisms involved in cortical plasticity occasionally required several seconds to take effect. ${ }^{8}$ Cortical reorganisation following changes in sensory input is a process which can develop in several successive steps, and as a result with different latencies according to the attributes of the images, such as colour, and movement. ${ }^{9}$

The perceived asymmetry was more readily recognised in the shoulders than in the face. This could be due to the fact that, in the affected visual field, the shoulder image corresponded to areas with wider defects than did the image of the lower part of the face. It may also be easier to evaluate the width of the shoulders than that of the face, which has an irregular shape and shows greater interindividual variability. Another reason may be that all borders of the visual field defect were included in the image of the shoulders, but not of the face. However, and in our opinion, this was most probably related to the fact that cortical remapping was more pronounced in the periphery of the visual field than in its centre, a phenomenon that has been well described using artificially induced scotomas. ${ }^{9}$

Neurophysiological observations of remapping in the visual cortex following bilateral lesions in the retina of adult cats and monkeys are of particular relevance to our understanding of cortical plasticity. ${ }^{2}$ Cortical changes occurring immediately after the retinal lesions include an increase in size of receptive fields and a shift in their location. Weeks following the retinal injury, cortical alterations presumably involve also changes in efficacy of pre-existing synapses and sprouting. ${ }^{10}$ As a result, following cortical rearrangement, the retinotopic map is modified, with a shift of the cortical representation to the part of the retina surrounding the lesion.

The idea that filling in was related to the expansion of receptive fields in the visual cortex was supported using artificially induced scotomas in cats and monkeys. ${ }^{811}$ In such a condition, it was found that expansion characteristics of receptive fields vary according to the areas of the visual cortex. In areas V2 and V3, the process occurred after only a few seconds of fixation, a time span that was comparable with that required by human subjects to perceive filling in of the masked area. In contrast, the receptive field of cells in area V1 expanded following several minutes' exposure to the stimulus.

Few studies have been devoted to spatial distortion following an altered pattern of sensory input. An important investigation was conducted by Kapadia et al with normal subjects. ${ }^{3}$ These authors showed that, using artificially induced scotomas, a shift was observed, pulling the perceived images surrounding the scotoma by a few minutes of arc toward the centre of the masked area. The shift increased with eccentricity from the fovea. It has been postulated that spatial distortion may also be related to expansion of receptive fields in the visual cortex. $^{3}$

In a study involving right homonymous paracentral scotomas, we found that affected individuals showed a slight but significant underestimation of distances from the central fixation point to dots located in areas surrounding the scotoma. ${ }^{4}$

In the phenomenon of shoulder narrowing experienced by the patients described in our report the shift in perceived image seemed greater than that measured in the above studies, which involved dots or bars, ${ }^{34}$ and in which the shift varied markedly according to eccentricity and the type of stimulus. This sug- 
gested that involvement of different structures in the cortex might result in different magnitudes of shift. In primates, the size of the receptive field increases along the pathway from V1 to V4, and from V4 to the inferior temporal cortex. ${ }^{12}$ Moreover, in higher visual functions, such as shape recognition, contextual influence probably takes place over larger areas of the visual cortex than does the more elementary processing of simple images, such as isolated dots or bars. This might also explain the fact that distortion was only better perceived when human shapes were considered than with other images.

The visual changes noted by our patients may share some features with alterations in visual assessment of sizes, called dysmetropsia, that have been described following posterior cerebral lesions. Transient dysmetropsia has been reported mainly as a manifestation of temporal lobe seizure, ${ }^{13}$ or during migraine episodes. ${ }^{14}$ It then affects either the entire visual field or the image visualised at the time of the event. Exceptionally, dysmetropsia has been noted to affect only parts of the visual field, as a rule in association with other visual changes, including metamorphopsia. ${ }^{15}{ }^{16}$ Brust and Behrens ${ }^{17}$ observed a patient who experienced transient visual disturbances, in which the right side of a person's face seemed to melt, "like clocks in a Dali painting". Objects other than faces were reportedly not involved. Computerised tomography showed a right posterior temporal translucency.

Permanent dysmetropsia after focal lesions has rarely been noted. Ebata et al observed a patient who claimed that the right side of faces - that is, the side viewed by the patient in his left hemifield, appeared smaller than the other side. ${ }^{18}$ Image distortion was restricted to faces, and the visual field was normal. Computerised tomography revealed a small haemorrhage in the right retrosplenial region.

In another study, Cohen et al reported an apparent reduction in size of objects when presented in one hemifield, in two patients with posterior cerebral damage. ${ }^{19}$ The left hemifield was affected in the first patient, who also demonstrated a variety of other visual disturbances including prosopagnosia, simultanagnosia, and difficulty in perceiving depth, proportions, and symmetry. Spatial distortion was also noted, but the visual field was normal on Goldmann perimetry. A right watershed infarct was located at the boundary between the territories of the middle and the posterior cerebral arteries. The second patient showed a right visual field defect described as a partial right inferior homonymous fascicular deficit, sparing the central 15 degrees. Other visual symptoms included simultanagnosia and right optic ataxia. This patient had suffered a bilateral occipitoparietal infarct. Cohen et al suggested that alterations in visual assessment of sizes could be related to changes in cells of the area V4, which had been shown to be tuned to the length or to the width of visual stimuli. ${ }^{20}$ Moreover, removal of this area was found to result in impairment of size discrimination. ${ }^{21}$
Thus, in the report by Cohen et al, field defects were noted but not taken into account. In the first patient, a left homonymous defect was initially present. Although, based on the results of Goldmann perimetry, the defect was apparently resolved at the time dysmetropia was experienced, it cannot be ruled out that a scotoma was still present but missed at the evaluation. In the second patient, a persisting homonymous defect was observed. With both patients, field defects were located on the side where distortion occurred. In the discussion, no correlation was made between field defects and dysmetropsia.

In our opinion, this association of field defects and spatial distortion deserves attention. This suggestion is supported by the clinical observation that, in both our patients, the image around the scotoma was perceived as pulled toward the gap in the visual field. Moreover, the neurophysiological findings mentioned above, related to cortical remapping taking place after focal deafferentation, and related perceptual filling in and shifts in spatial perception, provide a sound explanation for such an association.

Although this phenomenon was found in two successive patients suffering from homonymous paracentral scotomas, there is no evidence that it is invariably present in subjects with such visual field defects. It might depend upon the site and size of the lesion. Using white noise field campimetry in postchiasmal visual pathway lesions, it has recently been shown that the extent of filling in varies according to the site of the lesion. ${ }^{22}$ Moreover, in all our patients, homonymous paracentral scotomas happened to be right sided. It is uncertain whether right sidedness of the field defects was of any importance in the occurrence of the completion phenomena described in this study. In the study by Cohen et al, dysmetropsia was experienced in the left hemifield in one patient, whereas with the other patient it occurred on the opposite side. ${ }^{19}$ If the spatial distortion reported in their paper is similar in nature to that described here, one can conclude that this perceptual phenomenon is not related to right sidedness of the lesion.

Homonymous paracentral scotomas are not always readily recognised, even when they cause visual disturbances, such as in reading. Their presence can escape the subject's notice because of the completion phenomenon, which prevents the perception of any gaps in the affected visual field. They can also escape the attention of examiners because they are easily missed during routine visual field screenings, either by finger counting tests or routine automated programmes that include a limited number of test locations. As a result, when suspecting such defects, asking patients about asymmetry in perceived shoulder size might provide a useful clinical clue. A positive answer also suggests the presence of a completion phenomenon. This study was supported by the Swiss National Fund for Sci-
entific Research, grant nos 3200-040780.94/1, 3200entific Research, grant nos 3200-040780.94/1, 3200-
0409594.96 , and 4038-044081, and by the Sandoz Foundation for Research. 
1 Gilbert CD. Plasticity in visual perception and physiology. Curr Opin Neurobiol 1996;6:269-74.

2 Gilbert CD, Wiesel TN Receptive field dynamics in adult primary visual cortex. Nature 1992;356:150-2.

Kapadia MK, Gilbert CD, Westheimer G. A quantitative measure for short-term cortical plasticity in human vision. 7 Neurosci 1994;14:451-7.

4 Safran AB, Duret F, Mermoud C, et al. Altered perception of distances with homonymous paracentral scotomas. Vision Res 1996;36:S214.

5 Safran AB, Landis Th. Plasticity in the adult visual cortex Implications for the diagnosis of visual field defects and visual rehabilitation. Curr Opin Ophthalmol 1996;7(VI):5364 .

6 Ramachandran VS. Blind spots. Sci Am 1992;266:85-91.

7 Achard OA, Safran AB, Duret FC, et al. Role of the completion phenomenon in the evaluation of Amsler grid results. Am f Ophthalmol 1995;120:322-9.

8 De Weerd P, Gattass R, Desimone R, et al. Responses of cells in monkey visual cortex during perceptual filling-in of an in monkey visual cortex during perceptual

9 Ramachandran VS, Gregory RL. Perceptual filling-in of Ramachandran VS, Gregory RL. Perceptual filling-in of
artificially induced scotomas in human vision. Nature artificially induced

10 Das A, Gilbert CD. Long-range horizontal connections and their role in cortical reorganization revealed by optical recording of cat primary visual cortex. Nature 1995;375 $780-4$

11 Pettet MW, Gilbert CD. Dynamic changes in receptive field size in cat primary visual cortex. Proc Natl Acad Sci USA 1992;89:8366-70.

12 Desimone R, Ungerleider LG. Neural mechanisms of visual processing in monkeys. In: Boller F, Graftman J, eds.
Handbook of neuropsychology. Vol 2. Amsterdam: Elsevier, 1989:267-99.

13 Mullan S, Penfield W. Illusions of comparative interpretation and emotion. Arch Neurol Psychiatry 1959;81: 269-84.

14 Teuber HL, Battersby WS, Bender MB. Visual field defects after penetrating missile wounds of the brain. Cambridge: Harvard University Press, 1960

15 Mooney AJ, Carey P, Ryan M, et al. Parasagittal parietooccipital meningioma with visual hallucinations. $A m$ f Ophthalmol 1965;59:197-205.

16 Wilson SAK. Dysmetropsia and its pathogenesis. Trans Ophthalmol Soc UK 1916;36:412-44.

17 Brust JCM, Behrens MM. "Release hallucinations" as the major symptom of posterior cerebral artery occlusion: a report of 2 cases. Ann Neurol 1977;2:432-6.

18 Ebata S, Ogawa M, Tanaka Y, et al. Apparent reduction in the size of one side of the face associated with a small retrosplenial haemorrhage. $\mathcal{f}$ Neurol Neurosurg Psychiatry 1991;54:68-70.

19 Cohen L, Gray F, Meyrignac C, et al. Selective deficit of visual size perception: two cases of hemimicropsia. $\mathcal{F}$ Neurol Neurosurg Psychiatry 1994;57:73-8.

20 Desimone R, Schein SJ. Visual properties of neurons in area V4 of the macaque: sensitivity to stimulus form. $\mathcal{F}$ Neurophysiol 1987;57:835-68.

21 Schiller PH, Lee K. The role of the primate extrastriate area V4 in vision. Science 1991;251:1251-3.

22 Kolb M, Petersen D, Schiefer U, et al. Scotoma perception in white-noise-field campimetry and postchiasmal visual pathway lesions. Ger f Ophthalmol 1995;4:228-33. 